\title{
BMJ Open General practitioners' attitudes towards opioids for non-cancer pain: a qualitative systematic review
}

\author{
Rani Punwasi (D) , ${ }^{1}$ L de Kleijn, ${ }^{1}$ J B M Rijkels-Otters, ${ }^{1}$ M Veen, \\ Alessandro Chiarotto (D) ,' Bart Koes ${ }^{1,2}$
}

To cite: Punwasi R, de Kleijn L, Rijkels-Otters JBM, et al. General practitioners' attitudes towards opioids for non-cancer pain: a qualitative systematic review. BMJ Open 2022;12:e054945. doi:10.1136/ bmjopen-2021-054945

- Prepublication history and additional supplemental material for this paper are available online. To view these files, please visit the journal online (http://dx.doi.org/10.1136/ bmjopen-2021-054945).

Received 15 July 2021

Accepted 14 December 2021

Check for updates

(c) Author(s) (or their employer(s)) 2022. Re-use permitted under CC BY-NC. No commercial re-use. See rights and permissions. Published by BMJ.

${ }^{1}$ Department of General Practice, Erasmus Medical Center, Rotterdam, Zuid-Holland, Netherlands

${ }^{2}$ Centre for Muscle and Joint Health, University of Southern Denmark, Odense, Denmark

Correspondence to

Dr Rani Punwasi;

r.punwasi@erasmusmc.nl

\section{ABSTRACT}

Objectives Worldwide the use of opioids, both doctorprescribed and illicit, has increased. In most countries, opioids are first prescribed by general practitioners (GPS). Identifying factors that influence GPs' opioid prescription decision-making may help reduce opioid misuse and overuse. We performed a systematic review to gain insight into GP attitudes towards opioid prescription and to identify possible solutions to promote changes in the field of primary care.

Design and setting Systematic review of qualitative studies reporting GPs' attitudes towards opioid use in noncancer pain management.

Methods We searched Embase, Medline, Web of Science Core Collection, Cochrane, PsychInfo, Cumulative Index to Nursing \& Allied Health Literature (CINAHL) and Google Scholar. Two independent reviewers selected studies based on prespecified eligibility criteria. Study quality was evaluated with the Critical Appraisal Skills Programme checklist, and their results were analysed using thematic analysis. Quality of evidence was rated using the Grading of Recommendations Assessment, Development, and Evaluation-Confidence in the Evidence from Reviews of Qualitative research approach.

Results We included 14 studies. Four themes were established using thematic analyses: (1) GPs caught in the middle of 'the opioid crisis'; (2) Are opioids always bad? (3) GPs' weighing scale, taking patient-related and therapeutic relationship-related factors into account; and (4) GPs' sense of powerlessness-lack of alternatives, support by specialists and lack of time in justifying nonprescriptions.

Conclusion GP attitudes towards opioid prescribing for non-cancer pain are subject to several GP-related, patientrelated and therapeutic relationship-related factors. Raising GP and patient awareness on the inefficacy of opioids in chronic non-cancer pain management and providing non-opioid alternatives to treat chronic pain might help to promote opioid reduction in primary care. More research is needed to develop practical guidelines on appropriate opioid prescribing, tapering off opioid use and adopting effective communication strategies.

PROSPERO registration number CRD42020194561.Cite Now

\section{Strength and limitations of this study}

- To the best of our knowledge, this is the first review on this topic conducted by professionals working directly in primary care.

- We performed an analysis on the quality of the studies, as well as their relative contributions to the findings.

- Study screening and data extraction were conducted independently by two authors, with a third author mediating any disagreements.

- Most studies were performed in the USA making generalisability across countries limited.

- We only included publications written in English and in Dutch.

\section{INTRODUCTION}

Worldwide we are seeing a trend in increased opioid prescribing. ${ }^{1-3}$ The number of opioidrelated deaths and hospitalisations is also increasing. ${ }^{23}$ Opioids are commonly prescribed in the management of moderate-to-severe noncancer pain, in particular by general practitioners (GPs). ${ }^{245}$ In the past two decades, the number of opioid prescriptions by GPs has increased substantially. ${ }^{67}$ In the Netherlands, for example, GPs are responsible for approximately $75 \%$ of first opioid prescriptions and $90 \%$ of repeat prescriptions. ${ }^{89}$

Opioids can reduce acute and palliative pain, but have been shown to be ineffective for managing chronic non-cancer pain. ${ }^{10} 11$ Opioids are associated with side-effects like constipation, dizziness, falls and delirium. Additionally, using opioids can lead to opioid tolerance, dependence and even addiction; it is partly this addictive nature of opioids that has led to an increase of prescription opioid use disorder. ${ }^{12}{ }^{13}$ Worldwide, hospital admissions related to opioid use have increased in past years. ${ }^{14}$ In the USA, more than $4 \%$ of the adult population currently misuse prescription opioids, and the number of opioidrelated deaths per year increased sixfold 
between 1999 and 2017. ${ }^{1516}$ While this 'opioid crisis', as it is often called in the USA, is not comparable with the increase in opioid misuse in Europe, opioid prescription rates are nonetheless increasing and opioid-related hospitalisations and deaths are concerning. ${ }^{17-20}$ It is of utmost importance to decrease inappropriate opioid prescription rates.

The National Institute for Health and Care Excellence (NICE) guidelines explicitly ask doctors to refrain from opioid prescriptions for primary and secondary chronic pain (pain lasting $>3$ months) and recommend instead the use of conservative treatment options with no or very few side effects, such as exercise. ${ }^{21}$ In the UK, an Opioid Expert Working Group has been installed to address the increase of opioid use and misuse. This group has come with multiple recommendations that should inform patients about the risk of opioid dependence and addiction. ${ }^{22}{ }^{23}$ In the Netherlands, GP guidelines currently limit recommendations for strong opioids to restoring functional capacity in acute pain and to taper off as soon as possible. ${ }^{24}$ The Foundation for Pharmaceutical Statistics, an institute collecting prescription rates in the Netherlands, reported a $6 \%$ decrease in opioid prescriptions in 2019 compared with 2018, the first reduction seen after years of growth. ${ }^{17}$ Despite these modest positive signs, more action is needed to further decrease opioid prescriptions in the coming years.

Several systematic reviews elucidated multiple factors influencing GP opioid prescriptions. ${ }^{25}{ }^{26}$ However, conclusions were based on studies published before 2019 and the authors of these reviews lacked clinical experience in primary care. Commonly, guidelines and protocols in general practice are developed by the discipline itself in order to capture the 'richness of texture experienced in family practice'. ${ }^{27}{ }^{28}$ Since our review team mainly consists of GPs, or professionals involved in primary care research, we believe our clinical experience will generate a deeper level of understanding which may initiate practical changes in clinical practice that can address the increase of prescription opioid use disorder. Therefore, the aims of this study are to gain insight into GPs' attitudes, and the barriers and facilitators influencing GPs' opioid prescription practices, and to identify possible strategies to promote opioid reduction in primary care and to reduce the harm associated with opioid misuse.

\section{METHODS}

\section{Protocol registration}

This study followed the Enhancing Transparency of Reporting the Synthesis of Qualitative research (ENTREQ) framework. ${ }^{29}$ The ENTREQ framework is a validated method which offers guidance for researchers and reviewers to improve the reporting of synthesis of qualitative research. We prospectively registered our protocol in PROSPERO (ID CRD42020194561). online supplemental file 1

\section{Search strategy and study screening}

We searched Embase, Medline, Web of Science Core Collection, Cochrane, PsychInfo, Cumulative Index to Nursing \& Allied Health Literature (CINAHL) and Google Scholar for articles reporting GP attitudes on opioids prescription for non-cancer pain. Databases were searched from their inception date up to 17 September 2021 for articles written in English or Dutch. The search terms are presented in online supplemental table S1. All articles yielded were exported into Endnote $\mathrm{X} 7,{ }^{30}$ and duplicates were removed. Two reviewers (RP and LdK) independently reviewed titles and abstract. The same reviewers assessed full texts for inclusion. Finally, RP and LdK compared, discussed and reconciled their included articles with a third reviewer (AC). We identified qualitative studies describing GP attitudes or perspectives towards opioids prescription for non-cancer pain. We only extracted data attributed to GPs.

\section{Data extraction and analyses}

Two reviewers (RP and LdK) independently extracted the following data: author/year, title, study location, sample characteristics, research aim, data collection and analysis method, key themes and author conclusions. A thematic approach as described by Thomas and Harden ${ }^{31}$ was used to synthesise findings from the primary studies. First, two independent reviewers (RP and LdK) extracted line by line text including participants' quotations and findings of the original authors, and coded the text within an Excel sheet. Second, the same two reviewers (RP and LdK) independently developed descriptive themes by looking for similarities and differences among codes. These descriptive themes were discussed and refined into one thematic code book. Finally, a third reviewer (JBMR-O) re-examined this thematic code book. Disagreement was discussed until consensus was reached and the coding structure was adapted where necessary.

\section{Quality assessment}

To assess the methodological quality of each included study, two reviewers (RP, LdK) independently completed the Critical Appraisal Skills Programme checklist for qualitative research, which consists of 10 questions that evaluates method, credibility and the relevance of the study. ${ }^{32}$ Discrepancies between reviewers were discussed with a third reviewer (MV) until consensus was reached. We used the Grading of Recommendations Assessment, Development, and Evaluation-Confidence in the Evidence from Reviews of Qualitative research (GRADE-CERQual) approach to categorise confidence in the evidence into the following categories: good, minor, moderate or major concerns. ${ }^{32}$ The GRADE-CERQual covers four domains: (1) 'Methodological limitations' concern the conduct of each primary study; (2) 'Relevance' is the extent to which the primary studies are applicable to the review; (3) 'Adequacy of data' evaluates the overall richness and quantity of evidence; (4) 'Coherence' considers how well the findings are grounded in the primary studies. ${ }^{33}$ 


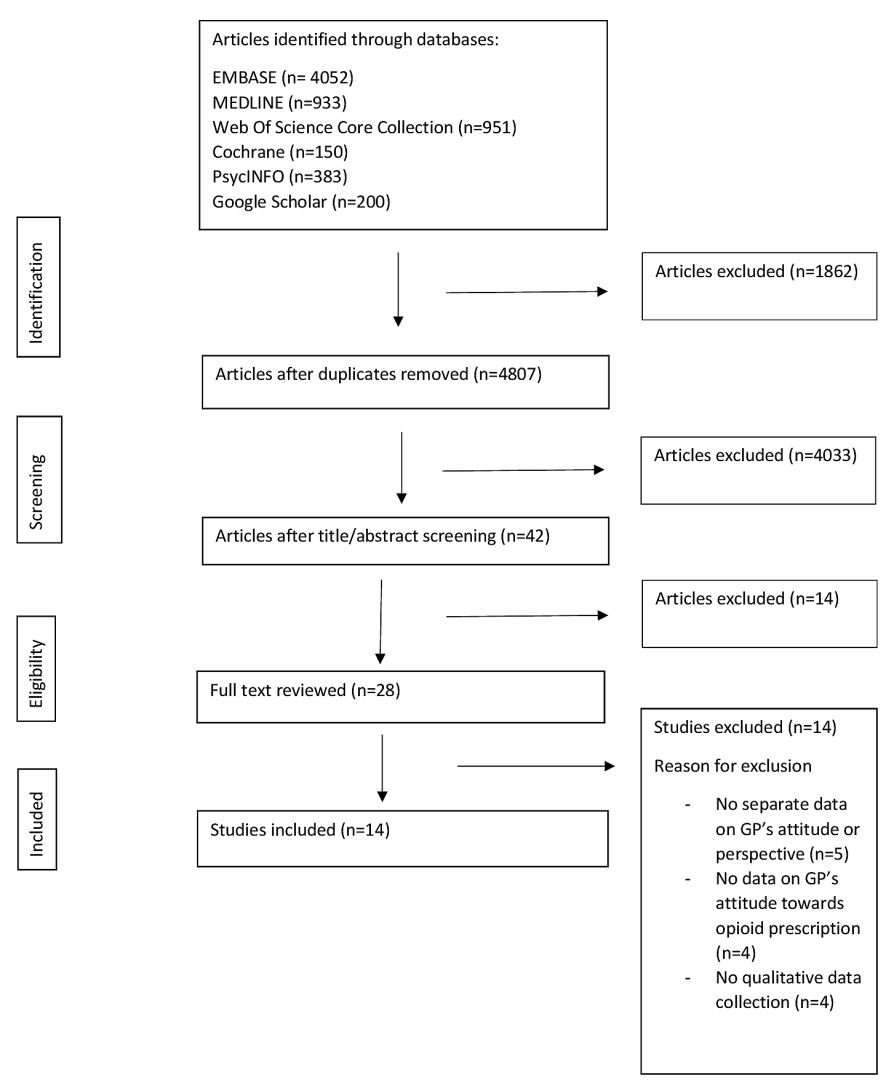

Figure 1 Preferred Reporting Items for Systematic Reviews and Meta-Analyses flow chart of article identification and selection. GP, general practitioner.

\section{Patient and public involvement}

There was no patient or public involvement in this review.

\section{RESULTS}

\section{Included articles}

Database searches resulted in 4807 unduplicated, potentially relevant articles (figure 1). After review of abstracts and titles, we selected 28 articles for full-text double screening. In total, 14 studies were included (table 1). ${ }^{34-47}$ The sample size ranged from 5 to 27 GPs. Five studies included solely GPs, ${ }^{37} 38414244$ and remaining studies also interviewed other primary care providers (PCP). In the USA, the term PCP is used for physicians providing primary care and consists of family doctors, internists, paediatrics, geriatrics, gynaecologists and nurse practitioners and physician assistants. ${ }^{48}$ For the current study, we only included family doctors. Nine studies were performed in North America, one study in Australia ${ }^{44}$ and the remaining four in Europe. ${ }^{39} 414546$

\section{Methodological quality assessment}

One study ${ }^{35}$ was appraised as moderately valuable, since no clarification was given on how the study sample was selected (table 2). The overall assessment of all but one (sub) theme was rated as high or moderate confidence (table 3).

\section{Thematic analysis}

Four main themes were constructed and further subdivided into several subthemes (online supplemental table S2). The four main themes were: (1) GPs caught in the middle of 'the opioid crisis'. (2) Are opioids always bad? (3) GP's weighting scale. (4) GP's sense of powerlessness. These themes are narratively explained based on data from the included articles and accompanied with quotations from their original studies (table 4).

\section{GPs caught in the middle of 'the opioid crisis' GP's duty to treat pain}

As healers, GPs desire to relieve patient's pain. ${ }^{37} 3842$ The subjective nature of pain complicates this mandate. ${ }^{37} 39$ GPs interviewed by Desveaux et $a l^{p^{78}}$ and Goodwin and Kirkland $^{42}$ stated that before the opioid crisis, it was believed that chronic pain was often undertreated. Some GPs found that analgesics other than opioids were seldom sufficient for chronic pain. ${ }^{37} 39$ Some GPs considered the patient as an undoubtable expert of their pain and considered it their job to address and eliminate pain. ${ }^{37} 38$ GPs from Desveaux et $a l^{\beta 7}$ reported that patients expect chronic pain to reach to zero. A range of emotional and psychosocial components contribute in maintaining chronic pain, making these expectations unrealistic. ${ }^{38} 42$ These GPs pleaded for more public awareness and education among patients regarding their pain. ${ }^{37}$

\section{GP's duty towards society at large}

Because of the well-known addictive character of opioids, some GPs reported a stigma in prescribing opioids. ${ }^{40} 41$ While some felt that the negative attention was unfair, others acknowledged the role that physicians have played in contributing to the opioid crisis. ${ }^{38}$ GPs emphasised and acknowledged their gatekeeper role in fighting the opioid crisis. ${ }^{34-47}$ However, because pain is subjective, some GPs doubted their medical decisions and at times created feelings of guilt that they might be undertreating their patients. ${ }^{37} 3940$ GPs felt caught between the desire to effectively treat pain and the societal obligation to decrease opioid prescriptions in order to reduce harm.

\section{Are opioids always bad?}

\section{Effectiveness and side-effects}

Several GPs stated that prescribing pain medication was based on a delicate balance between effective pain relief and possible side-effects. ${ }^{35}{ }^{36}$ In this matter, individualised prescribing is essential especially in elderly and patients with comorbidities. ${ }^{37} 41$ When restoring functional capacity and improving quality of life, GPs interviewed by Tong et $a l^{47}$ reported that the benefits of opioids at times outweighed the risks in chronic pain management. Several GPs' prescribing decisions were affected by possible side effects such as falls, drowsiness, constipation or nausea. ${ }^{41445}$ A small subset of selfdescribed 'militant' GPs avoided opioid prescription in patients with non-cancer due to limited indications and benefits. ${ }^{37}{ }^{38}$ GPs interviewed by Esquibel and Borkan ${ }^{40}$ 


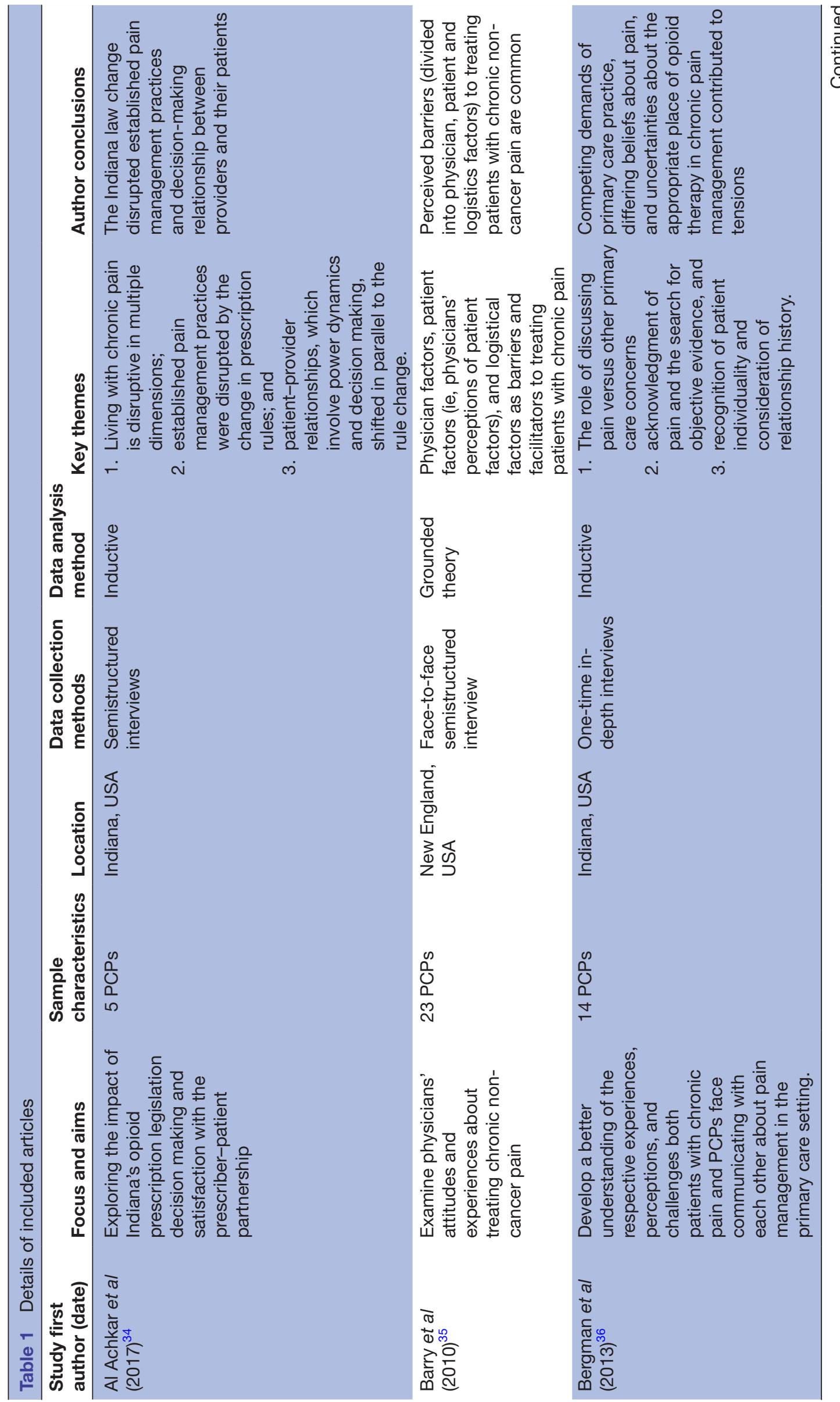




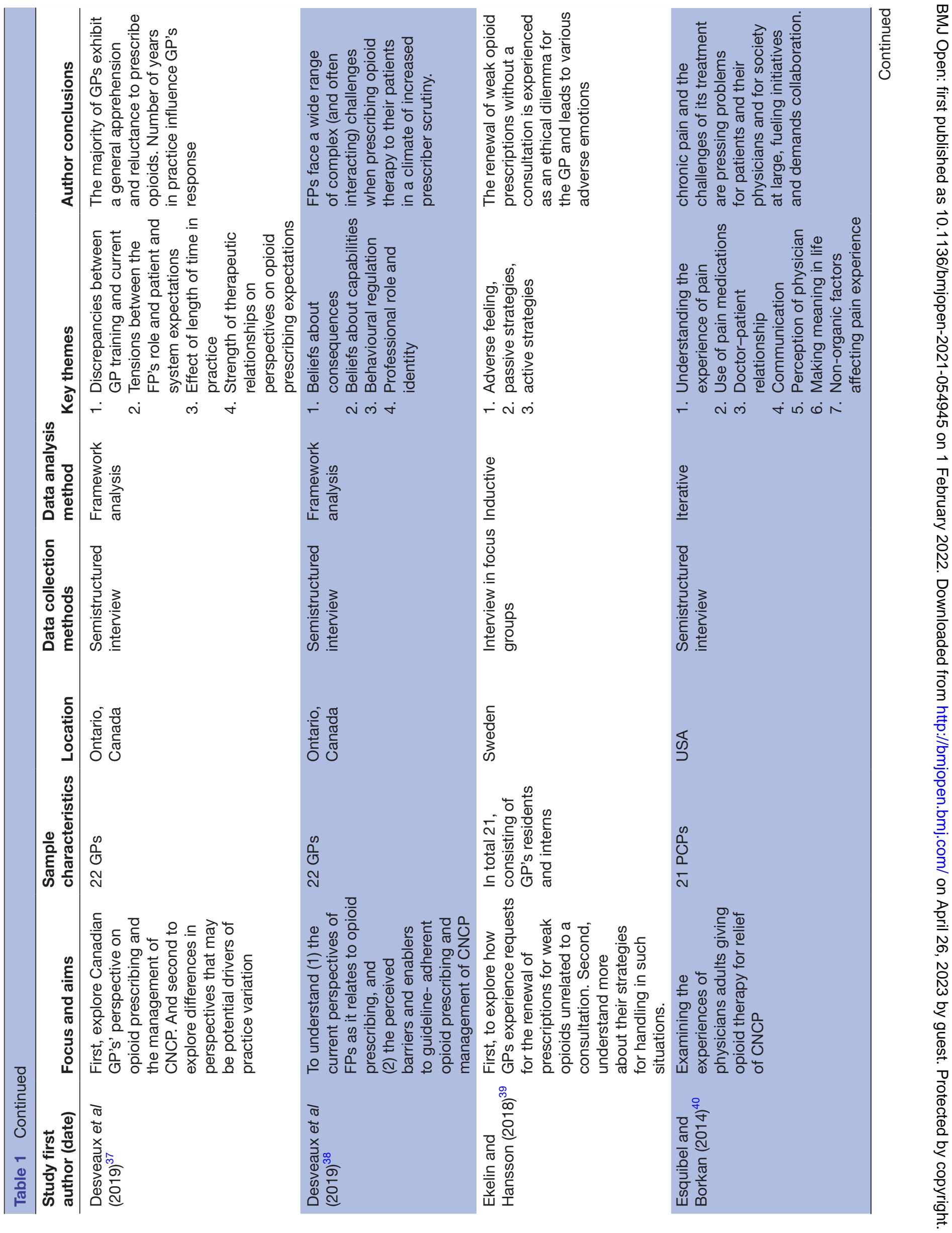




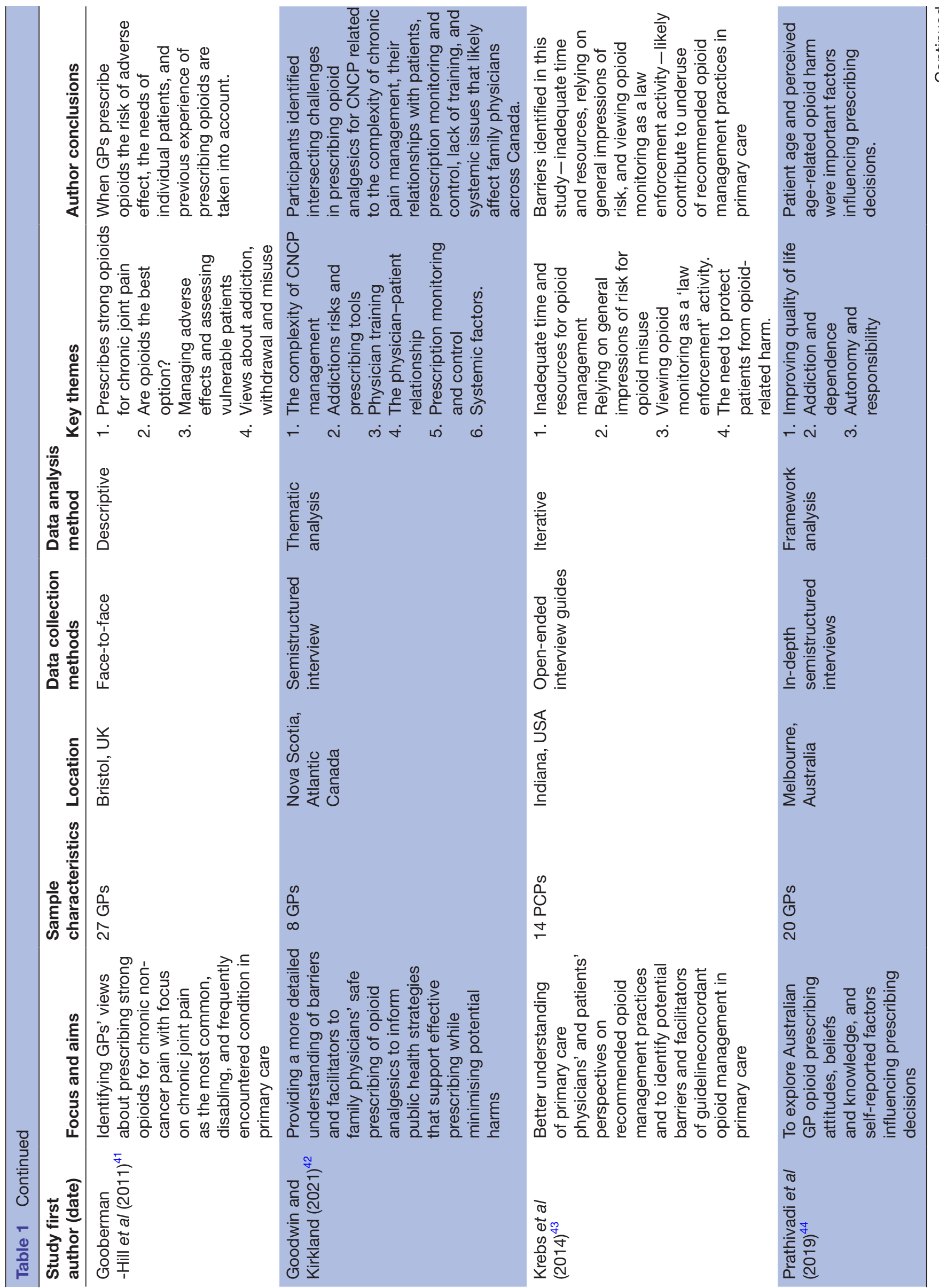

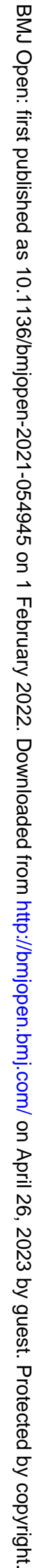




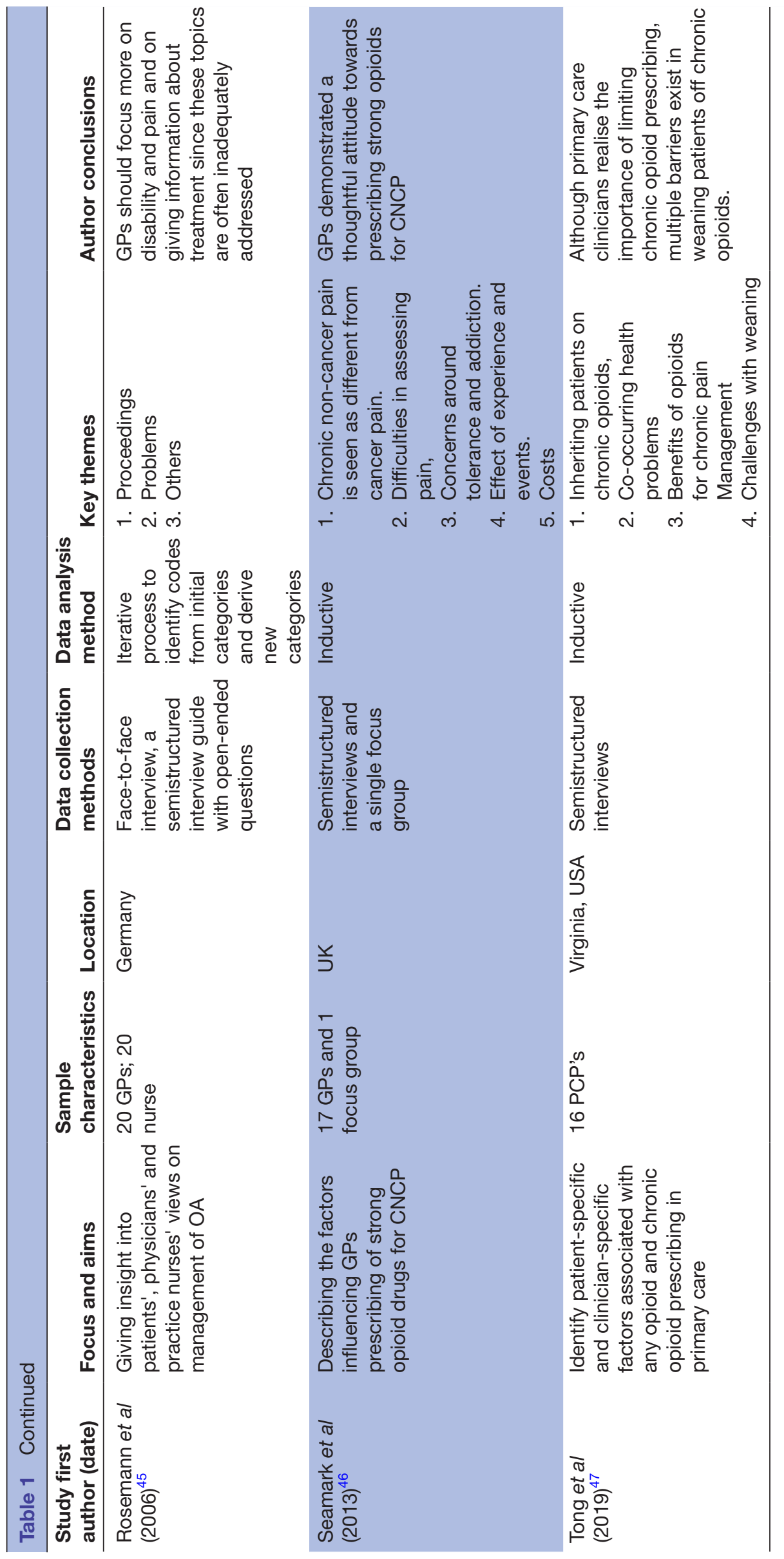

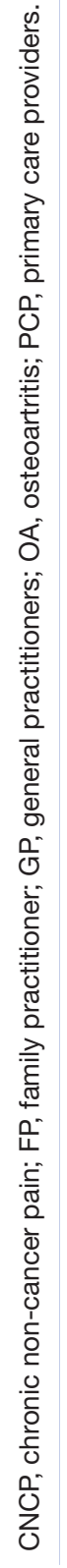




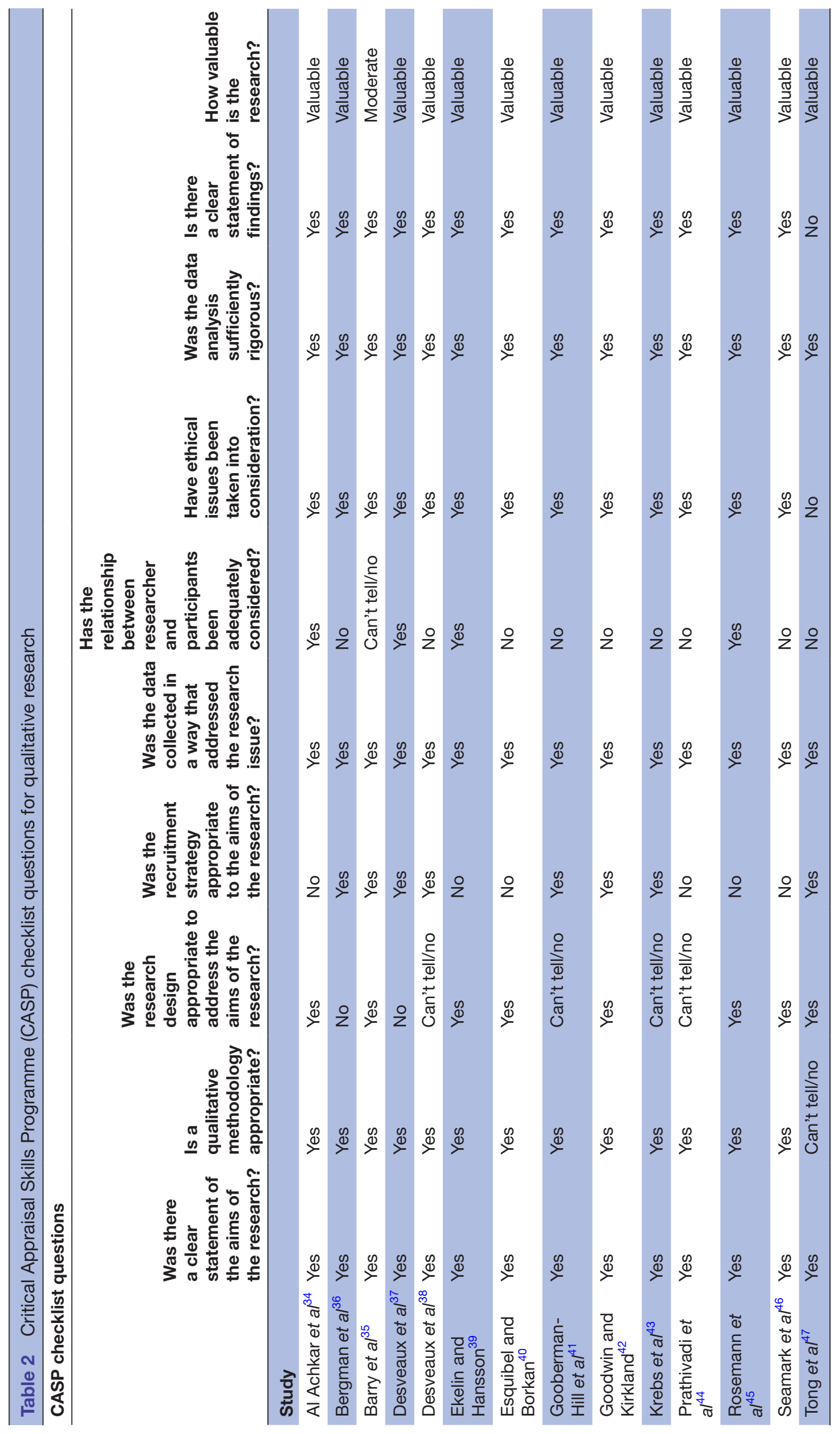


Table 3 Grading of Recommendations Assessment, Development, and Evaluation-Confidence in the Evidence from Reviews of Qualitative research framework

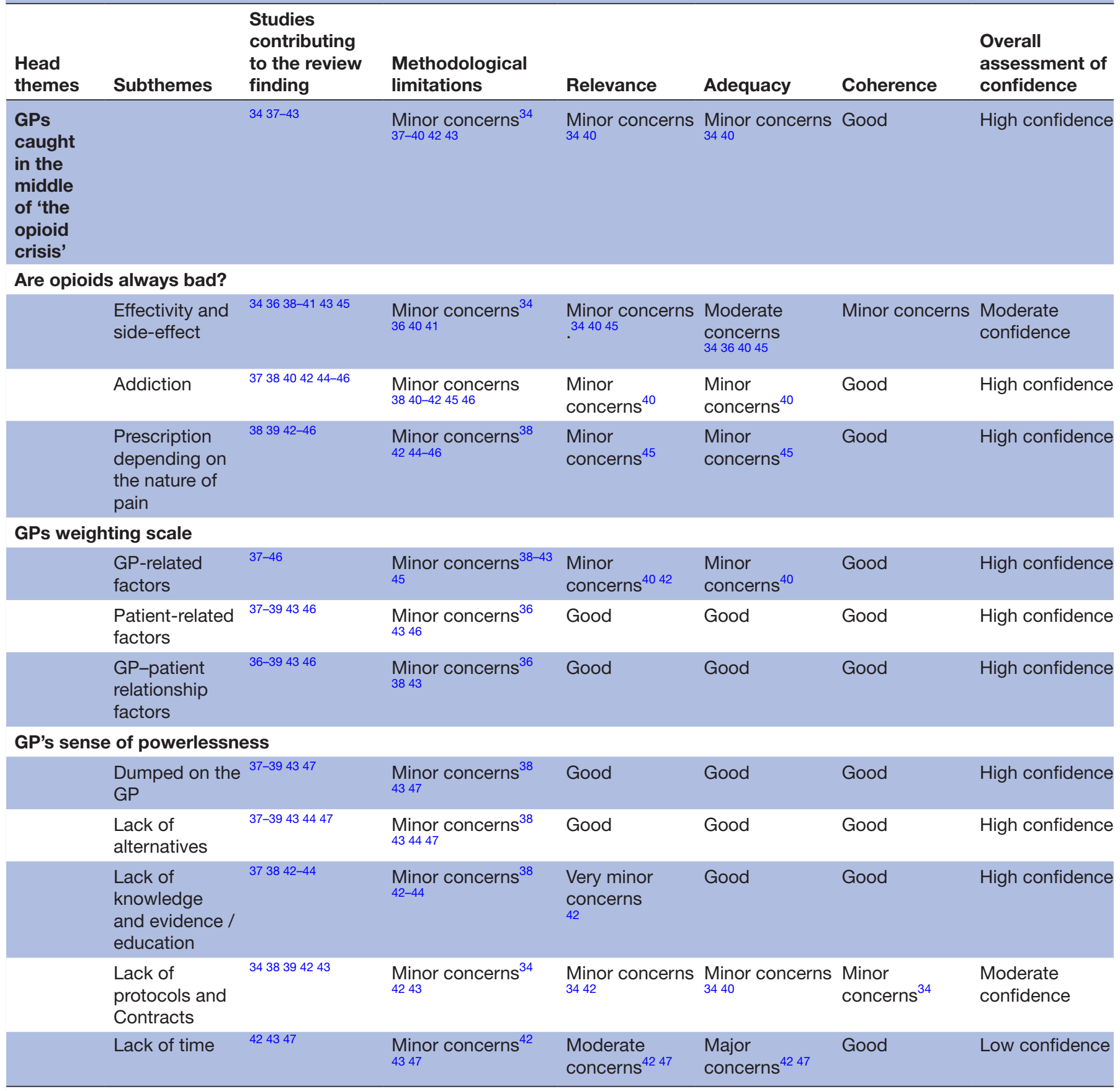

GP, general practitioner.

agreed with this statement and claimed that opioids lack evidence for long-term effectiveness and ultimately cause unwanted side effects. However, some GPs considered weak-acting or short-acting opioids acceptable for chronic non-cancer pain. ${ }^{39}$ GPs reported that the efficacy of weak or short-acting opioids differed largely. Some felt more comfortable prescribing short-acting instead of long-acting opioids because this gave them a sense of control. ${ }^{38}$ While others believed short-acting opioids increased the likelihood of break-through pain. ${ }^{38} 42$ GP's experience regarding the effectiveness of several types of weak opioids also influenced their preference. ${ }^{3941}$

Addiction

Growing knowledge on the addictive nature of opioids has made physicians reluctant to prescribe them. ${ }^{46}$ However, some GPs described addiction and misuse as a concern that should be dealt with, but should at the same time not be a barrier for prescribing opioids. ${ }^{41}{ }^{46}$ GPs interviewed by Seamark et $a t^{46}$ considered tolerance and the 
Table 4 Supporting Qualitative Data for Primary Themes

\section{Subthemes Quotations}

GPs caught in the middle of "the opioid crisis"

GP's duty to "I came out of school in [the 1990s]. At that point, we were undertreating chronic pain, so we were told. So we treat pain were quite gung-ho about not under-treating pain, and using opioids because they were supposedly safer than anti-inflammatories. And now, the pendulum has swung ... there's new evidence that it might actually not be doing them any good."37

"I feel like there should be some help for us in educating the public about keeping their use of opioids at the lowest possible level, it's your safety. That they shouldn't expect their pain to be zero because for chronic pain, it's probably not going to be possible to reach zero. If they can go from an 8 to a 5 , that's already pretty amazing. I feel like there should be a bit more public awareness and education."37

"As a primary care physician, you're being told to treat pain and to acknowledge patients' pain and to do something about it. And so, it's very difficult to walk that line. And all of those guidelines start with medications that are largely ineffective, for most people's pain."38

"I think the big problem for physicians is this sort of dual message that we keep getting -that physicians are part of the opiate problem and that we're undertreating pain. physician 7 "42

'You know this is helpful for you. This lets you get up and do your normal day, have your normal quality of life and without it you don't have [quality of life]. Do I have an alternative that works as well as this? Well, not really." 44

GP's duty "I think it's a very difficult balance, because there's certainly a lot of harm done by opioid prescribing by towards physicians. Physicians are at least responsible for controlling the supply of prescription opioids." 38

society at large "I think every doctor wants to do the right thing. I think $99.9 \%$, unless they're selling prescriptions or whatever. I think most doctors need more to do the right thing, because we didn't go into this profession to create drug addicts." 38

Are opioids always bad?

Effectiveness "Because some of us really like tramadol ... Others of us don't particularly like it at all. And it seems to cause and side- $\quad$ more side effects than codeine and stuff like that and people seem to feel sicker on it, and dizzier on it, and all effects sorts of stuff ... but it's fitting the drug to the patient." 41

"I feel like a change is not indicated at this time because she needs the medication in order to do her job and go to work and help her family, and it is working for her. She is overall low-risk for abuse. I don't feel compelled to make a change for her." 47

Addiction "I think there's a lot of unreasonable fears, the biggest one being addiction and I think it's a grossly, grossly overstated concern, addiction. In my practice l've yet to see the patient who was put on opiates for benign pain who is addicted. "46

"There's always the feeling that it's going to be more difficult for somebody to stop taking opioids or needing to take more, but it would depend on the personality" 46

"I'm always more concerned about people who have an abusive or abusing personality, or been abusive of other drugs in the past, particularly concurrent abuse of alcohol or other drugs." 46

Prescription "I have a bread and butter family medicine practice, cradle to grave. I probably prescribe about two patients a depending on week for acute pain, a limited prescription, and then I probably have about 30 to 35 patients who are on chronic the nature of opioids. Acute, it's not really a concern. I know my patients, I have a steady practice. So if I have a time limited pain prescription for a purpose that a person's pulled their back post-surgery, dental, you know, they'll get 10 to 20 and then never again, I'm not concerned about that."38

"I, personally, other than cancer patients or palliative care patients, have never started anyone on chronic opioids and I never would. I see no role for it in my practice."38

GP's weighing scale

GP-related "Um I suppose it's ... a bit of a vicious circle, it's lack of experience of getting people off the opioids ... The kind factors of fear that you're going to have someone hooked on it, which um I think is probably unfounded." ${ }^{41}$

But I don't really see much difference in the way that l'd use opioids [in chronic joint pain] to the way l'd use them in palliative care, I mean the principles are exactly the same of getting the dose right and ... titrating the dose with a liquid. ${ }^{41}$

'One of the reasons why I fear these medications so much or I hate them is because I don't like being in the situation where I have to now say something to this person. I fear how are they going to react? Are they going to get angry at me? Are they going to leave my care? ${ }^{44}$

"You just pick it up over the years, so I'm sure l've been moulded by the successes and the failures which have come my way in 27 years of general practice, yeah sure we all learn on the hoof, don't we?"46

"I'm not as slow to treat with opiates now as I was 30 years ago, and I'm sufficiently bigheaded that even if another doctor with the title consultant thought it was inappropriate l'd still go ahead and do it. If there was no other way of controlling someone's pain, and having discussed it with the patient, I'm prepared to do it."46

Patient-related "I think if someone's history shows that they have an addictive personality, whether it be street drugs, alcohol, factors smoking pot, whatever that theoretical concern is, but the patients l've used opiates for in noncancer are nearly always the elderly with joint pain and I don't have any concerns about them."46 
Table 4 Continued

\begin{tabular}{|c|c|}
\hline Subthemes & Quotations \\
\hline $\begin{array}{l}\text { GP-patient } \\
\text { relationship } \\
\text { factors }\end{array}$ & $\begin{array}{l}\text { "“I think the ones who trust me, knowing that I'm trying to help, won't leave angry." } \\
\text { "..., and that is exactly what they're doing. And sometimes they succeed. And then I feel bad because of it. I } \\
\text { think, now l've sort of failed as a doctor."39 } \\
\text { "But he kept coming for appointments and being aggressive about it. Verbally aggressive and the problem is, } \\
\text { he had genuine pain...I tried everything. It was very uncomfortable each visit because he is basically, in an } \\
\text { aggressive way, saying, I'm not helping [him] with the pain. - Physician 8"42 }\end{array}$ \\
\hline
\end{tabular}

GP's sense of powerlessness

Dumped on “It doesn't seem reasonable or right or medical. You can't really support this prescription that someone else has the GP

issued. You can't really take over this and stand for your own conviction"39

"These are prescription medications- they're coming from somewhere. It's us who are prescribing it, so we need to try and stop that. It might not be the GPs who are doing it, but we are by far the most accessible. We can try and address this issue. I see it as our duty to try and get them off these things that us a collective of doctors have actually hooked them onto [opioids] ${ }^{44}$

"She is seeing a psychiatrist, a pain specialist, an orthopedist, and a rheumatologist. She's got all of these people involved in her care but, for some reason, I'm the person who stuck with her pain med management and nobody is super-eager to touch that." 47

Lack of "I think the challenge, for me, is when you talk about decreasing, or trying to, patients kind of look at you and say alternatives

'But I still have pain. What do I do?' And often, there are not many other options. I don't have anywhere else [to send them] ... [so I] say yeah, I will do this for you. Sometimes you just don't have it. And I think, for me, that's the emotional part. ... You're caught between the college and trying to help this person, and the medical evidence and the lack of resources out there for people that should be there." 37

"I find it's just challenging because I don't know what else to offer. It's more that you feel bad for these people because they are in pain and even though these medications aren't good for pain really, I don't know what else to do for them."37

"Where's the support? Yeah, but where's the multidisciplinary approach? There aren't any community resources out there to help us." 38

Lack of "There isn't any patient support material. I just have the guidelines and I'm supposed to relay the information to knowledge them. And I'm relaying the information to a client that's very resistant to change. I have to be like a pharmaceutica and evidence / rep. I have to detail the patient. I have to get them to buy into the risk of the high doses. I don't have any support education material for that. I don't have any evidence or graphs or charts to present to the patient to say, 'Hey, if you're on a Benzo and a narcotic, you're at a higher risk of dying." 38

"...there had been no instruction whatsoever. I had no didactic training in pain management. Other than what you learn on the street. - Physician 2"42

Lack of "These are the rules. You know the rules. They're not my rules. Uh, this is the law and we can both agree that, you legislation and know, and those situations really practice in a way that's against the law. Hum, and so this makes it, it makes it appropriate more clear and objective and greatly reduces that kind of degree of emotional energy that was stressful prior to protocols and that. $^{34}$

contracts

Lack of time "In the community, [a family physician] might have a 5- or a 7- or 10- or 15-minute [appointment], and they totally have inadequate time to cover it. So, it can come up where you run out of time. - Physician 6"42

"The biggest problem in the whole thing is lack of time. Typically these are complex people with multiple problems, and you really could spend the whole appointment, more than 1 whole appointment, just talking about this [opioid agreement). I mean, we have all these reminders that we have to do, and all the scripts, and they're wanting a podiatry consult, and an eye consult, and you need to really sit down and go through a person's record, and really try to make a more rational decision. I take it very seriously. It's serious business. What if you do create an opiate problem for somebody? Because you're not being careful enough about it? ${ }^{43}$

GP, general practitioner.

possible requirement for more medication over the years when prescribing opioids. Some GPs believed long-acting opioids to have a higher likelihood for addictive potential and escalating doses. ${ }^{38}$ Many GPs feared addiction in patients with a history of substance misuse or patients with an 'abusive personality'. 3847

Prescription depending on the nature of pain

Some GPs considered opioids justified in chronic pain, while others considered it solely for terminal or palliative care. $^{37384647}$ GPs interviewed by Ekelin and Hansson expressed reluctance in prescribing opioids for psychosomatic illnesses. ${ }^{39}$ Opioid prescription was viewed as an overtreatment of osteoarthritis by several GPs. ${ }^{45}$

\section{GP's weighing scale}

GP-related factors

GP expertise plays a pivotal role in opioid prescription decision-making. A strong therapeutic relationship together with the number of years in practice made GPs 
feel more confident with their prescription decisions. ${ }^{38}$ Previous experience with opioid prescription and opioidspecific training was also mentioned as facilitators to feel more confident in prescribing opioids. ${ }^{41}{ }^{44}$ GPs also reported increased confidence in opioid prescription decision-making when they had worked in addiction centres or treated patients in a palliative care setting. ${ }^{38}$ Two studies showed that older and more experienced male doctors felt more confident in repeating weak opioid prescriptions. ${ }^{3941}$ GPs who lacked experience in tapering off opioids, felt less confident to prescribe opioids. ${ }^{41}$ Some GPs reportedly believed that refusing opioids or tapering off opioids would tempt patients to use illegal drugs instead. ${ }^{39}$ Some GPs with previous conflicts with patients regarding opioids avoided these analgesics 'as a mechanism to avoid challenging conversations' ${ }^{38}$ Moreover, prevailing standards on opioids and prescription behaviour among coworkers influenced GPs' prescription behaviour. ${ }^{3841}$

\section{Patient-related factors}

GPs reported patient age as an important factor in decision-making. ${ }^{46}$ Negative side-effects were considered more problematic in elderly patients than the potential for addiction. In contract, GPs considered opioids as a last resort in young adults due to the potential for addition. ${ }^{46}$ Improving social relationships and housing conditions were considered more important aspects than prescribing stronger medications. ${ }^{44}$ GPs interviewed by Seamark et $a t^{46}$ were reluctant to prescribe opioids in patients with a history of misuse or psychiatric illness. Some GPs expressed more confidence prescribing opioids for patients reluctant to receive opioid treatment compared with patients who demanded opioids because of fear of addiction. ${ }^{38}$

\section{GP-patient relationship factors}

Several GPs stated 'knowing the patient' facilitates decision-making in prescribing opioids. ${ }^{38}$ GPs declared that long-standing therapeutic relationships made it easier to decide whether or not to start opioids or to renew a prescription. GPs relied on patient's pain presentation for opioid prescription. However, in case of opioid prescriptions patients might not always be the most trustworthy partner. ${ }^{39}$ According to the GPs, the subjective nature of pain further enhanced the feeling of mistrust between the GPs and their patients. Some GPs described using a gut feeling in deciding to prescribe opioids. ${ }^{43}$ The potential loss of a doctor-patient relationship was a major concern for GPs when declining to prescribe opioids. ${ }^{38}$ GPs worried that they would be perceived as lacking empathy if they refused to prescribe opioids. Nonetheless, they acknowledged their responsibility to consider dependence and addiction. Many GPs considered talking about opioid treatment with patients to be a major source of conflict. ${ }^{384244}$ Some GPs even felt manipulated by their patients when discussing pain treatment. ${ }^{38}$

\section{GP's sense of powerlessness}

Dumped on the GP

GPs reported that specialists are more likely to prescribe opioids and do not do their due diligence in addressing the opioid crisis. ${ }^{38}$ They report feeling that the management of opioids is often 'dumped on the GP'. ${ }^{37} 38$ GPs reported feeling uncomfortable in renewing opioids when they disagreed on the indication or if they did not receive a clear handover on when and how to taper off. ${ }^{36} 424447$ Some GPs stood firm and refused renewal as they found it their responsibility to get their patients off of opioids. ${ }^{44}$ Yet other GPs stated they sometimes prescribed renewals to avoid difficult conversations with their patients. ${ }^{39} 42$

\section{Lack of alternatives}

GPs claimed to have a lack of alternatives when managing chronic pain, particularly in older patients. GPs reported that non-pharmacological options like regular physical activity, psychotherapy and physiotherapy were often rejected by patients. ${ }^{44}$ GPs reported reasons for not referring to specialised pain centres or private specialists included long waitlists, lack of affordability and likelihood that these referrals would end in opioid prescription anyhow as. ${ }^{42}{ }^{44}$ GPs interviewed by Desveaux $e t a l^{38}$ wanted a more interdisciplinary approach for chronic pain management. GPs reported that an impaired kidney function and contraindications made other pharmacological options limited. ${ }^{47}$

\section{Lack of knowledge and evidence/education}

GPs considered conversations about opioids to be difficult and to create tension in the GP-patient relationship. ${ }^{3742}$ Some GPs wanted more patient support material to educate patients about opioid treatment. In the absence of specialised training (ie, chronic pain management or addictions training), GPs felt less equipped to engage in conversations on opioids, and were thus more likely to adhere to current opioid prescription guidelines. ${ }^{3842}$

\section{Lack of legislation and appropriate protocols and contracts}

Some GPs desired clear legislation to guide and justify their therapy. ${ }^{3442}$ Others reportedly felt that current opioid protocols were too limited for use in practice and that there was not enough focus on providing alternatives. ${ }^{38}$ Some GPs stated that adhering to opioid guidelines interfered with their duty as a 'healer'. ${ }^{38}$ GPs' negative experiences with protocols and guidelines reduced adherence. ${ }^{38}$ Some GPs stated that a lack in appropriate protocols in tapering dosage resulted in avoiding opioid prescription. ${ }^{37}$ Several GPs did not know how to follow the recommended opioid management guidelines (such as drug screening and contracts) and stated to not use protocols as often as they should. ${ }^{43}$

\section{Lack of time}

GPs reported to be frustrated by a perceived lack of time with patients, particularly when needing to justify to the patient the denial of an opioid prescription. ${ }^{42} 46$ 


\section{DISCUSSION}

\section{Principal findings}

In this systematic review, we identified four main themes on GP attitudes towards opioid pain management: (1) GPs caught in the middle of the opioid crisis. (2) Are opioids always bad? (3) GPs weighing scale. (4) GP's sense of powerlessness. GP attitudes towards opioid prescribing for non-cancer pain are subject to several GP-related, patient-related and therapeutic relationship-related factors. The subjective nature of pain places GPs in a split position of being a healer but also a gatekeeper in the opioid crisis. The ongoing 'zero tolerance' trend in experiencing pain has led to a more liberal approach in prescribing opioids among some GPs. Some GPs consider opioids justified for (chronic) non-cancer pain management if functional capacity and quality of life improve, while others find opioids to have limited indication or benefit in these patients. GPs differed in age, experience, working place and GP-patient relationship, which may have influenced their attitudes. GPs who lacked experience in tapering off opioids felt less confident in opioid prescribing and were therefore less likely to prescribe opioids. Opioid prescription behaviour among coworkers also influenced prescription behaviours. Most GPs stated that knowing the patient facilitated decision-making in prescribing opioids. The potential loss of a doctorpatient relationship was a major concern for GPs when declining to prescribe opioids. GPs stated that current guidelines are too general and do not properly address the problems they face in daily clinical practice. Lack of support by specialists and access to multidisciplinary pain centres frustrated GPs.

As demonstrated by our findings and related studies, ${ }^{25} 26$ the addictive nature of opioids is widely recognised in primary care and is one of the factors that make GPs refrain from prescribing opioids. Importantly, the ineffectiveness of opioids was not reported as a major factor to GPs in determining their opioid prescription patterns. Even when the ineffectiveness of opioids was recognised, GPs felt morally obliged to alleviate pain and still considered opioids as a last resort in chronic pain. This reflects the lack of alternatives and knowledge on how to effectively address chronic non-cancer pain. This review underscores the importance of educating GPs on effective strategies in relieving chronic non-cancer pain, but also on conversation techniques to engage in difficult conversations with patients about pain and pain acceptance. That said, broadening GP knowledge alone will not be sufficient, raising awareness among patients is also important. Patients should be well informed about the impact of chronic pain and that a pain reduction to zero is often impossible. Patients have to realise that opioids are not 'the Holy Grail'. Developing patient support materials may help to create awareness among patients. Improvement of the communication between GPs and specialists is also much needed. As GPs, we recognise the powerlessness felt when after hours of motivational talks, discussing the inappropriateness of opioid use with our patients, we decide to refer them to a pain centre for alternative pain treatment, which then results in patients returning to our care with opioid prescriptions with no further explanation or communication.

GPs in this review complained how current guidelines are too general and do not properly address the problems they face in daily clinical practice. A recent Australian qualitative review analysed GP attitudes towards interventions aimed at reducing opioid prescriptions by GPs and proposed that codesigning guidelines with end-users (GPs) might influence their success. ${ }^{49}$ Although previous publications ${ }^{2526}$ underline the importance of the development of new guidelines, we believe that underlining the importance of GP's involvement in developing these guidelines is also much needed.

The included studies were conducted in six different countries, with different healthcare systems, but despite this, themes identified were broadly consistent. Although the themes and bottlenecks GPs face were similar, different healthcare systems may require other strategies to address their unique problems. We believe the abovementioned recommendations such as educating GPs and patients, improving collaboration between GPs and specialists and developing guidelines for GPs by GPs will work across different healthcare systems. However, solutions should be adapted to fit local needs and demands. Encouraging country-specific changes at health insurance policy level should be part of local opioid reduction strategies. Recently, several Dutch universities (Radboud University in Nijmegen, Utrecht Medical Centre in Utrecht, Leiden Medical Centre in Leiden and the Erasmus Medical centre in Rotterdam), joined forces to decrease inappropriate opioid use in primary care. ${ }^{50}$ Together they investigate the causes and consequences of opioid use in the Netherlands and also aim to influence policy level changes.

\section{Strengths and limitations}

A strength of our study is that GPs' perspectives on opioid treatment for non-cancer pain were synthesised by a review team of mainly GPs using a transparent and robust methodology to generate new and comprehensive themes reflecting data across different geographical settings. We acknowledge that our direct involvement in primary care might be a source of bias; however, we believe that our backgrounds enable a deeper level of understanding of this topic. This review has included eight studies ${ }^{34} 37-39444547$ that were not included in the two most recent reviews on this topic. Five studies were excluded in this systematic review because these also included data on other primary care givers such as nurse practitioners or doctor's assistants and the data regarding GPs could not be separated. By excluding these studies, we are aware that we might have lost some potentially useful data. Not each study has equally contributed to the presented data. In Rosemann et $a l,{ }^{45}$ only one paragraph was dedicated to GPs attitudes towards opioid prescriptions. In Al Achkar et al, ${ }^{34}$ only two GPs were included making the data extraction minimal. 
Moreover, a majority of the studies were performed in the USA making generalisability limited.

\section{CONCLUSION}

This review demonstrates the difficulties encountered by GPs in treating (chronic) non-cancer pain and refraining from opioid prescription: a zero-tolerance policy towards pain by both doctors and patients; a wish for strong doctor-patient relationships with a fear of difficult conversations; a lack of knowledge and protocols on effective strategies to treat (chronic) pain in primary care; a lack of time; and inadequate collaboration with, and guidance from, specialists. Our findings highlight that in order to promote appropriate opioid prescription in primary care and to reduce the harms associated with opioid misuse, future research is needed to develop practical guidelines on appropriate opioid prescribing, tapering off opioid use and adopting effective communication strategies not only for GPs but also fine-tuned by GPs.

Contributors RP and LdK made substantial contribution to the conception and/or design of the work. RP, LdK, JBMR- 0 and AC contributed to the acquisition, analysis and interpretation of data for the work. All authors provided input to drafting the work and/ or revising it critically and gave final approval of the version to be published. RP is guarantor.

Funding The authors have not declared a specific grant for this research from any funding agency in the public, commercial or not-for-profit sectors.

Competing interests None declared.

Patient consent for publication Not applicable.

Ethics approval This study does not involve human participants.

Provenance and peer review Not commissioned; externally peer reviewed.

Data availability statement № data are available.

Supplemental material This content has been supplied by the author(s). It has not been vetted by BMJ Publishing Group Limited (BMJ) and may not have been peer-reviewed. Any opinions or recommendations discussed are solely those of the author(s) and are not endorsed by BMJ. BMJ disclaims all liability and responsibility arising from any reliance placed on the content. Where the content includes any translated material, BMJ does not warrant the accuracy and reliability of the translations (including but not limited to local regulations, clinical guidelines, terminology, drug names and drug dosages), and is not responsible for any error and/or omissions arising from translation and adaptation or otherwise.

Open access This is an open access article distributed in accordance with the Creative Commons Attribution Non Commercial (CC BY-NC 4.0) license, which permits others to distribute, remix, adapt, build upon this work non-commercially, and license their derivative works on different terms, provided the original work is properly cited, appropriate credit is given, any changes made indicated, and the use is non-commercial. See: http://creativecommons.org/licenses/by-nc/4.0/.

\section{ORCID iDs}

Rani Punwasi http://orcid.org/0000-0002-6267-7709

Alessandro Chiarotto http://orcid.org/0000-0003-2350-9945

\section{REFERENCES}

1 De Conno F, Ripamonti C, Brunelli C. Opioid purchases and expenditure in nine western European countries: 'are we killing off morphine?' . Palliat Med 2005;19:179-84.

2 Fischer B, Jones W, Rehm J. Trends and changes in prescription opioid analgesic dispensing in Canada 2005-2012: an update with a focus on recent interventions. BMC Health Serv Res 2014;14:90.

3 Kelly JP, Cook SF, Kaufman DW, et al. Prevalence and characteristics of opioid use in the US adult population. Pain 2008;138:507-13.
4 The Royal Australian College of General Practitioners. Prescribing drugs of dependence in general practice. Part C1: opioids. East MelbourneVictoria: RACGP, 2017.

5 Ashaye T, Hounsome N, Carnes D, et al. Opioid prescribing for chronic musculoskeletal pain in UK primary care: results from a cohort analysis of the COPERS trial. BMJ Open 2018;8:e019491.

6 Verhamme KMC, Bohnen AM. Are we facing an opioid crisis in Europe? Lancet Public Health 2019;4:e483-4.

7 Weesie Y. Ook Nederlandse huisartsen schrijven vaker opioïden voor. Huisarts en Wetenschap 2018;61:10.

8 Brakel van. Verslag rondetafelconferentie VWS:gebruik opoïden, 2018.

9 Nivel. Available: https://www.nivel.nl/sites/default/files/bestanden/ Rapport_voorschrijven_opioiden.pdf

10 Jensen MK, Thomsen AB, Højsted J. 10-Year follow-up of chronic non-malignant pain patients: opioid use, health related quality of life and health care utilization. Eur J Pain 2006;10:423-33.

11 Tucker H-R, Scaff K, McCloud T, et al. Harms and benefits of opioids for management of non-surgical acute and chronic low back pain: a systematic review. Br J Sports Med 2020;54:664.

12 Benyamin R, Trescot AM, Datta S, et al. Opioid complications and side effects. Pain Physician 2008;11:S105-20.

13 Up-To-Date, pain control in the critically ill adult patient.

14 Häuser W, Schug S, Furlan AD. The opioid epidemic and national guidelines for opioid therapy for chronic noncancer pain: a perspective from different continents. Pain Rep 2017;2:e599.

15 Hancocks S. The opioid crisis in the USA. Br Dent J 2019;226:815.

16 Saha TD, Kerridge BT, Goldstein RB, et al. Nonmedical prescription opioid use and DSM-5 nonmedical prescription opioid use disorder in the United States. J Clin Psychiatry 2016;77:772-80.

17 Stichting Farmaceutische Kengetallen, 2020. Available: https://www. sfk.nl/publicaties/PW/2020/copy_of_scores-kwaliteitsindicatorenook-grafi-sch-weergegeven

18 van Amsterdam J, van den Brink W. The misuse of prescription opioids: a threat for Europe? Curr Drug Abuse Rev 2015;8:3-14.

19 Publications Office of the European Union. European monitoring centre for drugs and drug addiction European drug report 2018: trends and developments. Luxembourg, 2018.

20 Dart RC, Severtson SG, Bucher-Bartelson B. Trends in opioid analgesic abuse and mortality in the United States. N Engl J Med 2015:372:241-8.

21 NICE guideline. Chronic pain (primary and secondary) in over 16S: assessment of all chronic pain and management of chronic primary pain, 2021.

22 Pharmatimes. Available: http://www.pharmatimes.com/web_ exclusives/is the uk facing its own opioid crisis 1315

23 Uk government. Available: https://www.gov.uk/government/news/ opioid-expert-working-group-meets-at-mhra

24 Nederlandse Huisartsen Genootschap. NHG standaard pijn, 2018.

25 Kennedy M-C, Pallotti P, Dickinson R, et al. 'If you can't see a dilemma in this situation you should probably regard it as a warning': a metasynthesis and theoretical modelling of general practitioners' opioid prescription experiences in primary care. $\mathrm{Br} J$ Pain 2019;13:159-76.

26 Toye F, Seers K, Barker KL. Meta-ethnography to understand healthcare professionals' experience of treating adults with chronic non-malignant pain. BMJ Open 2017;7:e018411.

27 Jaye $C$. Doing qualitative research in general practice: methodological utility and engagement. Fam Pract 2002;19:557-62.

28 Burgers JS, Wittenberg J, Keuken DG, et al. Development of a research agenda for general practice based on knowledge gaps identified in Dutch guidelines and input from 48 stakeholders. Eur $J$ Gen Pract 2019;25:19-24.

29 Tong A, Flemming K, Mclnnes E, et al. Enhancing transparency in reporting the synthesis of qualitative research: ENTREQ. BMC Med Res Methodol 2012;12:181.

30 Thomson Reuters. endnote X7, 2014. Available: http://endnote.com/ product-details $/ x 7$

31 Thomas J, Harden A. Methods for the thematic synthesis of qualitative research in systematic reviews. BMC Med Res Methodol 2008;8:45.

32 Casp tool. Available: http://cfkr.dk/images/file/CASP\% 20instrumentet.pdf

33 Lewin S, Glenton C, Munthe-Kaas $\mathrm{H}$, et al. Using qualitative evidence in decision making for health and social interventions: an approach to assess confidence in findings from qualitative evidence syntheses (GRADE-CERQual). PLoS Med 2015;12:e1001895.

34 Al Achkar M, Revere D, Dennis B, et al. Exploring perceptions and experiences of patients who have chronic pain as state prescription opioid policies change: a qualitative study in Indiana. BMJ Open 2017;7:e015083. 
35 Barry DT, Irwin KS, Jones ES, et al. Opioids, chronic pain, and addiction in primary care. J Pain 2010;11:1442-50.

36 Bergman AA, Matthias MS, Coffing JM, et al. Contrasting tensions between patients and PCPs in chronic pain management: a qualitative study. Pain Med 2013;14:1689-97.

37 Desveaux L, Saragosa M, Kithulegoda N, et al. Family physician perceptions of their role in managing the opioid crisis. Ann Fam Med 2019;17:345-51.

38 Desveaux L, Saragosa M, Kithulegoda N, et al. Understanding the behavioural determinants of opioid prescribing among family physicians: a qualitative study. BMC Fam Pract 2019;20:59.

39 Ekelin E, Hansson A. The dilemma of repeat weak opioid prescriptions - experiences from swedish GPs. Scand J Prim Health Care 2018;36:180-8

40 Esquibel AY, Borkan J. Doctors and patients in pain: conflict and collaboration in opioid prescription in primary care. Pain 2014;155:2575-82.

41 Gooberman-Hill R, Heathcote C, Reid CM, et al. Professional experience guides opioid prescribing for chronic joint pain in primary care. Fam Pract 2011;28:102-9.

42 Goodwin J, Kirkland S. Barriers and facilitators encountered by family physicians prescribing opioids for chronic non-cancer pain: a qualitative study. Health Promot Chronic Dis Prev Can 2021;41:182-9.
43 Krebs EE, Bergman AA, Coffing JM, et al. Barriers to guidelineconcordant opioid management in primary care--a qualitative study. $J$ Pain 2014;15:1148-55.

44 Prathivadi P, Barton C, Mazza D. Qualitative insights into the opioid prescribing practices of Australian GP. Fam Pract 2020;37:412-7.

45 Rosemann T, Wensing M, Joest K, et al. Problems and needs for improving primary care of osteoarthritis patients: the views of patients, general practitioners and practice nurses. BMC Musculoskelet Disord 2006;7:48.

46 Seamark D, Seamark C, Greaves C, et al. Gps prescribing of strong opioid drugs for patients with chronic non-cancer pain: a qualitative study. Br J Gen Pract 2013;63:e821-8.

47 Tong ST, Hochheimer CJ, Brooks EM, et al. Chronic opioid prescribing in primary care: factors and perspectives. Ann Fam Med 2019;17:200-6.

48 Healthline. Available: https://www.healthline.com/find-care/articles/ primary-care-doctors/what-is-a-primary-care-physician\#types-of-pcps

49 Prathivadi P, Luckett T, Barton C, et al. General practitioner attitudes towards systems-level opioid prescribing interventions: a pooled secondary qualitative analysis. Aust J Gen Pract 2021;50:309-16.

50 Erasmus MC. Available: https://www.erasmusmc.nl/en/research/ project/mis-opioids 